\title{
Mapping by spatial predictors exploiting remotely sensed and ground data: A comparative design-based perspective
}

\author{
Piermaria Corona ${ }^{\text {a,* }}$, Lorenzo Fattorini ${ }^{\text {b }}$, Sara Franceschi ${ }^{\text {b }}$, Gherardo Chirici ${ }^{c}$, Fabio Maselli ${ }^{\text {d }}$, Luca Secondi ${ }^{\mathrm{e}}$ \\ a Consiglio per la ricerca e la sperimentazione in agricoltura, Forestry Research Centre (CRA-SEL), Arezzo, Italy \\ ${ }^{\mathrm{b}}$ Department of Economics and Statistics, University of Siena, Siena, Italy \\ c DiBT, University of Molise, Pesche, Italy \\ d IBIMET-CNR, Firenze, Italy \\ e Department for Innovation in Biological, Agro-food and Forest Systems (DIBAF), University of Tuscia, Viterbo, Italy
}

\section{A R T I C L E I N F O}

\section{Article history:}

Received 11 February 2014

Received in revised form 16 May 2014

Accepted 17 May 2014

Available online $\mathrm{xxxx}$

\section{Keywords:}

Forest mapping

Tessellation stratified sampling

Universal kriging

Cokriging

Locally weighted regression

$k$-Nearest neighbor predictor

Simulation

\begin{abstract}
A B S T R A C T
This study was designed to compare the performance - in terms of bias and accuracy - of four different parametric, semiparametric and nonparametric methods in spatially predicting a forest response variable using auxiliary information from remote sensing. The comparison was carried out in simulated and real populations where the value of response variable was known for each pixel of the study region. Sampling was simulated through a tessellation stratified design. Universal kriging and cokriging were considered among parametric methods based on the spatial autocorrelation of the forest response variable. Locally weighted regression and $k$-nearest neighbor predictors were considered among semiparametric and nonparametric methods based on the information from neighboring sites in the auxiliary variable space. The study was performed from a design-based perspective, taking the populations as fixed and replicating the sampling procedure with 1000 Monte Carlo simulation runs. On the basis of the empirical values of relative bias and relative root mean squared error it was concluded that universal kriging and cokriging were more suitable in the presence of strong spatial autocorrelation of the forest variable, while locally weighted regression and $k$-nearest neighbors were more suitable when the auxiliary variables were well correlated with the response variable. Results of the study advise that attention should be paid when mapping forest variables characterized by highly heterogeneous structures. The guidelines of this study can be adopted even for mapping environmental attributes beside forestry.
\end{abstract}

(C) 2014 Elsevier Inc. All rights reserved.

\section{Introduction}

Sound management of forest areas requires accurate information regarding the extent, condition and productivity of natural resources. Estimation of these variables is an agreed objective of environmental monitoring programs at a variety of spatial scales. Distinctively, wallto-wall forest variable maps are important information sources to guide forest and environmental management decisions (e.g., Corona, Chirici, McRoberts, Winter, \& Barbati, 2011; Davis, Johnson, Bettinger, \& Howard, 2001).

Traditionally, data on forests and forestry are collected by means of user-driven sampling procedures, in the context of forest inventories (Corona \& Marchetti, 2007). In turn, forest inventories are usually performed using probabilistic sampling schemes in which a set of plots is selected from the investigated area in accordance with some spatial design. It should be noticed that the purely random scheme is likely to produce unsuitable voids in the study area. To ensure that plots are evenly spread over the region, stratified schemes are preferred. One such

\footnotetext{
* Corresponding author.
}

scheme involves tessellation of the study area by means of regular polygons of equal size (e.g., the national forest inventories of USA and Italy), each of them containing at least a portion of the study area, and then the random selection of one point in each of these polygons. This scheme is usually referred to as the tessellation stratified sampling (TSS).

Remote sensing data acquired from aerial and space platforms can support modern forest inventories as $i$ ) auxiliary information to improve the precision of inventory estimates, and ii) auxiliary information for forest variable mapping (Corona, 2010; McRoberts \& Tomppo, 2007). In most situations, remotely sensed data from multispectral optical or radar images or metrics calculated from airborne laser scanning (ALS) are available for all the pixels of the investigated area, in contrast with the values of forest variables which are known only for the sampled portion of the area (McRoberts, Cohen, Naesset, Stehman, \& Tomppo, 2010).

Several statistical techniques employing information coupled from remotely sensed imagery and ground data are available for predicting forest variables for the unsampled portion of the area and thus deriving a wall-to-wall map. All these techniques involve the use of spatial predictors based on a set of assumptions generating the probability distribution of the forest variable over the study area and, at the same time, 